\title{
Diversidad, abundancia y frecuencia de argonautas (Cephalopoda: Argonautidae), en la dieta de peces pelágicos de importancia comercial en Oaxaca, México
}

Diversity, abundance and frequency of argonauts (Cephalopoda: Argonautidae) in the diet of pelagic fishes of commercial value in Oaxaca, Mexico

\section{María del Carmen Alejo-Plata ${ }^{*}$, Sairi Sarai León-Guzmán², Román Díaz-Polo ${ }^{3}$ y Ana María Torres-Huerta ${ }^{1}$}

\author{
'Instituto de Recursos, Universidad del Mar, Campus Puerto Ángel, Ciudad Universitaria, San Pedro Pochutla, Oaxaca, 70902, México \\ ${ }^{2}$ Posgrado en Ecología Marina, Universidad del Mar, Campus Puerto Ángel, Ciudad Universitaria, San Pedro Pochutla, Oaxaca, 70902, \\ México \\ ${ }^{3}$ Programa en Biología Marina, Universidad del Mar, Campus Puerto Ángel, Ciudad Universitaria, San Pedro Pochutla, Oaxaca, 70902, \\ México \\ *Autor corresponsal: plata@angel.umar.mx
}

\begin{abstract}
The argonauts are a family of pelagic octopus with wide distribution in tropical and subtropical waters of the world, whose presence is notable in the diet of various species of pelagic fish with commercial value. The present study assessed the diversity, relative abundance and frequency of these cephalopods in the gastric content of 7 species of fish (4 teleosts and one elasmobranch), captured by the artisanal fishery of the state of Oaxaca, Mexico. We examined 778 stomachs obtained in monthly samplings during 2016 and 2017. The Argonauts were the most significant prey among the octopuses (98\%) and the second most important group of cephalopods (35\%) after the squids (42.5\%). A total of 273 females for 3 species Argonauts were analyzed: Argonauta nouryi (45.6\%), A. $\operatorname{argo}(20.8 \%)$, y $A$. hians (33.6\%). Two types were observed of the shells of $A$. nouryi: type $A$. nouryi (16.3\%) and type $A$. cornuta $(29.3 \%)$. Thus, we emphasize the usefulness of the studies of feeding in pelagic predatory fishes for the capture and the bioecological study of the Argonauts in the eastern tropical Pacific.
\end{abstract}

Key words: Pelagic octopods, argonauts, diet of pelagic fishes, Gulf of Tehuantepec

Resumen.- Los argonautas son una familia de pulpos pelágicos con amplia distribución en aguas tropicales y subtropicales del océano mundial, cuya presencia es notable en la dieta de diversas especies de peces pelágicos con valor comercial. En el presente estudio se evaluó la diversidad, abundancia relativa y frecuencia de este grupo de cefalópodos presentes en el contenido gástrico de 7 especies de peces (4 teleósteos y un elasmobranquio), capturados por la pesquería artesanal del estado de Oaxaca, México. Se examinaron 778 estómagos obtenidos de muestreos mensuales durante 2016 y 2017. Los argonautas fueron las presas más representativas del grupo octopoda (98\%) y el segundo grupo más importante de cefalópodos (35\%) después de los calamares (42,5\%). Un total de 273 hembras correspondientes a 3 especies de argonautas fueron analizadas: Argonauta nouryi (45,6\%), A. argo (20,8\%), y A. hians (33,6\%). Se observaron dos tipos en las conchas de $A$. nouryi: tipo $A$. nouryi $(16,3 \%)$ y tipo $A$. cornuta $(29,3 \%)$. De este modo, se destaca la utilidad de los estudios de alimentación en peces depredadores pelágicos para llevar a cabo la captura y el estudio bioecológico de argonautas en el Pacífico tropical oriental.

Palabras clave: Pulpos pelágicos, argonautas, dieta de peces pelágicos, Golfo de Tehuantepec

\section{INTRODUCCIÓN}

Los argonautas son organismos enigmáticos y fascinantes (Nesis 1977), cuyo conocimiento sobre su biología y ecología en el Pacífico oriental tropical es escaso. La familia Argonautidae constituye un grupo de octopodos de ambientes epipelágicos. Las hembras tienen una concha blanca translúcida y quebradiza que funciona como una cámara de ovoposición para la protección de los huevos. Es construida de calcita-Mg a partir de una glándula especializada que se encuentra en los extremos distales del primer par de brazos dorsales (Smith et al. 2012). Los argonautas macho presentan un dimorfismo sexual extremo, son muy pequeños respecto a la hembra (1:12 la longitud del manto de la hembra), nunca producen concha (Nesis 1977) y habitan la zona pelágica.

El desconocimiento del cuerpo blando de las hembras argonautas llevó a un sistema taxonómico para la familia basado únicamente en la concha. Sin embargo, la enorme plasticidad en la forma de la concha entre especies ha causado confusión a los taxónomos a lo largo de los años, 
lo que ha dado lugar a 50 especies consideradas hoy como sinonimias (Finn 2013). La familia Argonautidae está compuesta por un único género, Argonauta, con 4 especies: Argonauta nouryi Lorois, 1852; Argonauta argo Linnaeus, 1758; Argonauta hians Lightfoot, 1786 y Argonauta nodosa Lightfoot, 1786 (Finn 2014). Se reconocen tres especies para México: $A$. argo y $A$. hians en el golfo de México (Salcedo-Vargas 1991); A. argo y A. nouryi en el océano Pacífico (Finn 2014).

Las hembras argonauta destacan como grupo importante en la dieta de peces óseos (atún, dorado, marlín y pez vela) y cartilaginosos (tiburón azul, bironche y sedoso), con distribución en el noreste del Pacífico de México, incluyendo el Golfo de California (Rosas-Alayola et al. 2002, Arizmendi et al. 2006, Cabrera-Chávez-Costa et al. 2010, Markaida \& Sosa-Nishizaki 2010, Tripp-Valdez et al. 2010, Hernández-Aguilar et al. 2012, Galván-Magaña et al. 2013, Olson et al. 2014, Osuna-Peralta et al. 2014, Duffy et al. 2015, Ortega-García et al. 2018). A pesar de la importancia de las hembras argonauta como alimento de diversas especies de peces, han sido escasamente estudiadas en el Pacífico mexicano, lo que podría deberse a lo raro de su captura con vida en su ambiente natural. Además, la concha al ser muy delicada se fragmenta fácilmente y su cuerpo carnoso es rápidamente digerido por los depredadores, como ha sido observado en otros cefalópodos (Olson \& Galván-Magaña 2002). Sólo los picos quitinosos resisten la digestión y mantienen su forma, siendo los más usados para la identificación de especies (Xavier \& Cherel 2009). Sin embargo, al no existir claves por especie la identificación se limita al nivel de género.

Por lo anterior, y con la finalidad de profundizar en el conocimiento del grupo de octópodos en el Pacífico mexicano, el presente estudio tiene como objetivo la evaluación de la abundancia y frecuencia de argonautas hembra dentro del contenido gástrico de 7 especies de peces pelágicos capturados por la pesca artesanal que se desarrolla en el litoral del estado de Oaxaca, México. Asimismo, determinar el valor de este tipo de análisis como método alternativo para la obtención de ejemplares de argonautas. De modo adicional, se entrega información sobre la relación peso total-longitud del manto, distribución de frecuencia de tallas y algunos aspectos reproductivos de los argonautas.

\section{MATERIALES Y MÉTODOS}

La zona de estudio corresponde a la costa de Oaxaca dentro del Golfo de Tehuantepec (Fig. 1), región que se caracteriza por su plataforma continental cuya amplitud máxima de $\sim 106,8 \mathrm{~km}$ (Ahumada-Sempoal, com. pers.) ${ }^{1}$. Se ubica a los $93-94^{\circ} \mathrm{W}$ y tiene una amplitud mínima de $\sim 17,8 \mathrm{~km}$ a los $95,5^{\circ} \mathrm{W}$ (NOAA-NCEI) ${ }^{2}$. Así también, en la zona se presentan lluvias de mayo a octubre y estiaje de noviembre a abril, con un periodo (mayo/junio) donde se forman tormentas tropicales y huracanes, que se llega a extender hasta octubre/noviembre (Reyes \& Mejía-Trejo 1991).

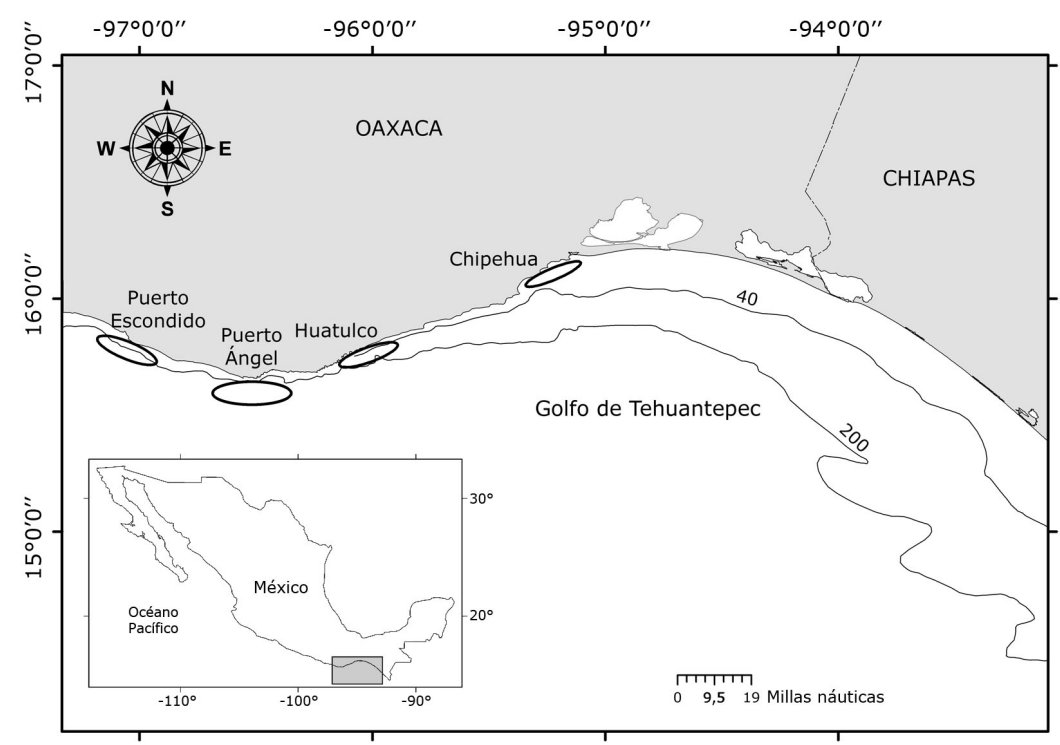

Figura 1. Localización del área donde se capturaron los peces. Los polígonos marcados representan las áreas de pesca y se indican las localidades de desembarco de la pesca artesanal / Location of the area where fish were caught. Doted polygons represent the fishing area and the landing locations of the artisanal fishing are indicated

\footnotetext{
${ }^{1}$ Miguel-Angel Ahumada-Sempoal, Universidad del Mar, Puerto Ángel,

San Pedro Pochutla, Oaxaca, México. <ahumada@angel.umar.mx>

${ }^{2}<$ https://www.ngdc.noaa.gov/mgg/global/etopo2.html>
} 
Es característico del Golfo de Tehuantepec, el arribo en noviembre y abril de vientos del Norte, también llamados "Tehuanos" con intensidades mayores $10 \mathrm{~m} \mathrm{~s}^{-1} \mathrm{y}$ duración típica de 2 a 6 días (Lavín et al. 1997, Romero-Centeno et al. 2003). Este fenómeno contribuye de forma significativa al incremento de la productividad primaria (clorofila- $a>1,0$ $\left.\mathrm{mg} \mathrm{m}^{-3}\right)$ y descenso de la temperatura $\left(\sim 17^{\circ} \mathrm{C}\right)$ en el golfo (Trasviña et al. 1995, Lluch-Cota et al. 1997).

El área de muestreo comprendió las principales zonas de desembarque de la pesca ribereña del estado de Oaxaca localizadas en Puerto Escondido, Puerto Ángel, Santa Cruz Huatulco y Bahía Chipehua (Fig. 1).Los equipos de pesca activos utilizados fueron curricanes, cuya especie objetivo es el barrilete Euthynnus lineatus (Kishinouye, 1920) y el atún aleta amarilla Thunnus albacares (Bonnaterre, 1788), la captura se efectuó a no más de $5 \mathrm{~km}$ de la costa. Además de boyas (palangre modificado) con las que se captura atún aleta amarilla, pez vela Istiophorus platypterus (Shaw, 1792) y el dorado Coryphaena hippurus Linnaeus, 1758, la pesca se realiza en aguas costeras a no más de $40 \mathrm{~km}$. En tanto que los equipos de pesca pasivos fueron palangres (cimbras) y redes de enmalle, usados para la pesca de tiburón, e incidentalmente también se captura pez vela y dorado; la pesca se realizó en aguas oceánicas a más de 50 $\mathrm{km}$ de la costa.

Se realizaron muestreos mensuales dentro del periodo de enero del 2016 a diciembre del 2017. Los peces de una submuestra del desembarco total fueron medidos. Para el pez vela se registró la distancia ojo-furca $(\mathrm{OF}, \mathrm{cm})$; para el dorado, barrilete negro, atún aleta amarilla y tiburón sedoso Carcharhinus falciformis (Müller \& Henle, 1839) la longitud furcal (LF, cm).

A partir de lo cual se obtuvieron un total de 778 estómagos, de los cuales $306(39,3 \%)$ correspondieron a E. lineatus $(\mathrm{LF}=35-65 \mathrm{~cm}), 206(26,5 \%)$ a $T$. albacares (50-85 cm), $123(15,8 \%)$ a C. hippurus $(\mathrm{LF}=20-145 \mathrm{~cm})$, $99(12,7 \%)$ a I. platypterus $(\mathrm{OF}=128-225 \mathrm{~cm})$ y $44(5,6 \%)$ a C. falciformis $(\mathrm{LF}=35-250 \mathrm{~cm})$.

Los estómagos fueron inmediatamente removidos de los peces y se mantuvieron en frío hasta llegar al laboratorio, donde se revisaron cuidadosamente. El examen de los contenidos gástricos se enfocó en la búsqueda de argonautas intactos y picos frescos, los cuales se preservaron en etanol al 70\% para su posterior determinación con base en los criterios de Finn $(2013,2014)$ que toman en cuenta como caracteres diagnósticos el número de lamelas por hemibranquia, la morfología de los aparatos reproductor y digestivo, la glándula de la tinta y el caecum. Para aquellos ejemplares sin concha o parcialmente digeridos, se utilizaron los picos para su determinación de acuerdo a Clarke (1986); los picos de argonauta acumulados fueron descartados, para evitar incluir organismos que posiblemente no se consumieron en el área de estudio. Los ejemplares completos, picos y conchas se depositaron como referencia en la Colección de Cefalópodos de la Universidad del Mar, en Puerto Ángel, Oaxaca, México.

La contribución total de los argonautas a la dieta de los depredadores se calculó como la suma del porcentaje en peso $(\% \mathrm{P})$, porcentaje en número $(\% \mathrm{~N})$ y porcentaje de frecuencia de ocurrencia $(\% \mathrm{FO})$, en relación con otras categorías de presa en los estómagos de cada depredador.

A los organismos preservados completos y sin rastros de digestión, se les midió la longitud dorsal del manto (LDM, mm) con un vernier digital (precisión 0,01 mm); el peso total (PT) se midió con una balanza digital $(0,01 \mathrm{~g})$. A cada organismo se le extrajo la masa bucal, misma que fue transferida a hipoclorito de sodio al $30 \%$ en agua destilada para limpiar los picos. Estos fueron enjuagados con agua destilada y almacenados en etanol al 70\% con unas gotas de glicerol para evitar resequedad. Se midió la longitud del capuchón en la mandíbula superior (UHL, en inglés) y en la inferior (LHL, en inglés). Estas dimensiones fueron elegidas debido a la facilidad de medición (Xavier \& Cherel 2009). Se usaron regresiones de mínimos cuadrados para evaluar las relaciones entre UHL o LHL (mm) y el peso $(\mathrm{g})$. Los datos fueron transformados a logaritmo natural (Ln) para cumplir con los supuestos de normalidad y homogeneidad de varianza (Zar 1999).

Por otra parte, se estimó la relación Peso total (PT)longitud dorsal del manto (LDM) a través de ecuación potencial PT $=a \mathrm{LDM}^{b}$. La ecuación fue linealizada por transformación logarítmica, donde $a$ representa la ordenada al origen y $b$ la pendiente de la ecuación. Para determinar si la pendiente $b$, es igual (crecimiento isométrico) o diferente (crecimiento alométrico) de 3 se aplicó una prueba de pendientes mediante el estadístico $t$-Student (Zar 1999).

\section{Resultados}

De los 778 estómagos revisados, $33 \%$ contenía algún tipo de alimento; de estos, $80 \%$ presentó argonautas. Los argonautas fueron la presa más frecuente en la dieta del pez vela $(\mathrm{FO}=$ $55,3 \%)$, seguidos del barrilete $(\mathrm{FO}=28,6 \%)$, el dorado $(\mathrm{FO}=$ $23,5 \%)$, el atún $(\mathrm{FO}=8,7 \%)$ y el tiburón sedoso $(\mathrm{FO}=5 \%)$ (Tabla 1). Se observaron argonautas completos y con las conchas intactas en las regurgitaciones del barrilete y pez vela (Fig. 2). 
Tabla 1. Porcentaje de abundancia (\%N), porcentaje peso estimado (\%P) y frecuencia de ocurrencia (\%FO) de argonautas encontrados como parte del contenido estomacal de pelágicos mayores capturados por la pesca artesanal en la costa de Oaxaca, México / Percentage of abundance $(\% \mathrm{~N})$ estimated weight percentage (P\%) and frequency of occurrence (\%FO) of Argonauts found as part of the stomach contents of larger pelagic fish caught by artisanal fishing off the coast of Oaxaca, Mexico

\begin{tabular}{|c|c|c|c|c|c|c|c|c|c|c|c|c|c|c|c|}
\hline \multirow{2}{*}{ Especie } & \multicolumn{3}{|c|}{$\begin{array}{l}\text { Istiophorus } \\
\text { platypterus }\end{array}$} & \multicolumn{3}{|c|}{$\begin{array}{l}\text { Coryphaena } \\
\text { hippurus }\end{array}$} & \multicolumn{3}{|c|}{ Euthynnus lineatus } & \multicolumn{3}{|c|}{ Thunnus albacares } & \multicolumn{3}{|c|}{$\begin{array}{l}\text { Carcharhinus } \\
\text { falciformis }\end{array}$} \\
\hline & $\% \mathrm{~N}$ & $\% \mathrm{P}$ & $\% \mathrm{FO}$ & $\% \mathrm{~N}$ & $\% \mathrm{P}$ & $\% \mathrm{FO}$ & $\% \mathrm{~N}$ & $\% \mathrm{P}$ & $\% \mathrm{FO}$ & $\% \mathrm{~N}$ & $\% \mathrm{P}$ & $\% \mathrm{FO}$ & $\% \mathrm{~N}$ & $\% \mathrm{P}$ & $\% \mathrm{FO}$ \\
\hline \multicolumn{16}{|l|}{ Octopodiformes } \\
\hline \multicolumn{16}{|l|}{ Argonautidae } \\
\hline Argonauta nouryi & 6,4 & 0,3 & 9,0 & 4,6 & 0,4 & 3,8 & 1,5 & 0,1 & 4,7 & 1,2 & $<0,1$ & 1,4 & 1,7 & $<0,1$ & 5 \\
\hline Argonauta hians & 13,2 & 0,5 & 18,6 & 9,5 & 0,8 & 7,9 & 3,0 & 0,2 & 9,6 & 2,6 & $<0,1$ & 2,9 & & & \\
\hline Total & 39,3 & 1,6 & 55,3 & 28,3 & 2,4 & 23,5 & 9,1 & 0,5 & 28,6 & 7,6 & $<0,1$ & 8,7 & 1,7 & $<0,1$ & 5 \\
\hline Octopodidae & & & & & & & 4,0 & 0,4 & 14,3 & 0,2 & 0,1 & 4,3 & & & \\
\hline \multicolumn{16}{|l|}{ Decapodiformes } \\
\hline
\end{tabular}

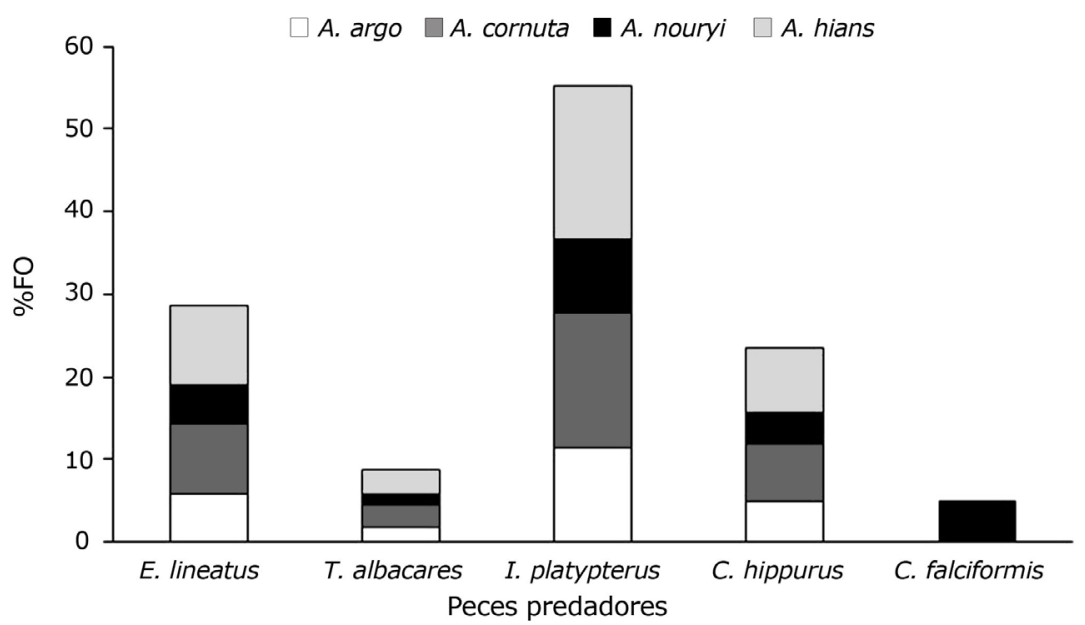

Figura 2. Frecuencia de ocurrencia de los argonautas presa en la dieta de los peces depredadores en el Pacífico sur de México / Frequency of occurrence of argonauts prey in the diet of predatory fishes in the South Pacific of Mexico

Los argonautas fueron la presa más significativa entre los octopoda $(98,0 \%)$ y el segundo grupo más importante de cefalópodos $(39,0 \%)$ después de los calamares Omastréfidos $(42,5 \%)$. En la mayoría de estómagos únicamente se encontraron picos.

Un total de 273 hembras correspondientes a 3 especies de argonautas fueron analizadas. Se observaron dos tipos en las conchas de Argonauta nouryi: tipo Argonauta nouryi $(\mathrm{n}=45 ; 16,3 \%)$ y tipo Argonauta cornuta $(\mathrm{n}=80 ; 29,3 \%)$ (Fig. 2). Las hembras de Argonauta argo $(\mathrm{n}=56 ; 20,8 \%)$ se encontraron sin restos de concha. Se reporta por primera vez la presencia de Argonauta hians ( $\mathrm{n}=92 ; 33,6 \%$ ) en el océano Pacífico tropical oriental (Tabla 2).

Argonauta nouryi (13,9-50,8 mm LDM, 24,1 $\pm 9,9 \mathrm{~mm}) \mathrm{y}$ A. cornuta (10,9-44,6 mm LDM, 24,3 \pm 7,3 mm) presentaron las mayores tallas seguidas de $A$. hians $(6,2$ a $41,1 \mathrm{~mm}$ LDM, 24,7 $\pm 6,01 \mathrm{~mm}$ ). Las hembras de Argonauta argo únicamente se registraron en tallas pequeñas, inmaduras y sin concha (11,6 a 18,9 mm LDM, 14,6 $\pm 3,1 \mathrm{~mm}$ ) (Fig. 3). 
Tabla 2. Intervalo de conteos e índices para hembras de Argonauta nouryi, Argonauta cornuta, Argonauta argo and Argonauta hians del Pacífico sur de México / Ranges of counts and indices for females of Argonauta nouryi, Argonauta cornuta, Argonauta argo and Argonauta hians from South Pacific of Mexico

\begin{tabular}{lcccc}
\hline & \multicolumn{4}{c}{ Formas Argonauta nouryi } \\
\cline { 2 - 5 } & A. nouryi & A. cornuta & A. hians & A. argo \\
\hline Número & 34 & 61 & 70 & 43 \\
Talla brazos & $2>4$ & $2>4$ & $2>4$ & $4>2$ \\
LDM (mm) & $13,9-24,1-50,8$ & $10,9-24,3-44,6$ & $6,2-23-41,1$ & $11,6-16,2-18,9$ \\
FB & $2 \geq 3 \geq 4$ & $2 \geq 3 \geq 4$ & $2 \geq 3>4$ & $4>2>3$ \\
GC & $13-16$ & $13-15$ & $9-13$ & $16-17$ \\
\#hectocotilo por hembra & $1-9$ & $1-6$ & $1-3$ & - \\
\#ventosas hecototilo & $58-60$ & $58-60$ & $37-44$ & 110 \\
Diámetro huevos (mm) & $0,6-1,0$ & --- & $39-42$ & -- \\
Diámetro embriones (mm) & $0,8-1,2$ & --- & $0,7-1,2$ & -- \\
\hline
\end{tabular}

LDM: Longitud dorsal del manto, FB: fórmula brazos, GC: número de lamelas por hemibranquia
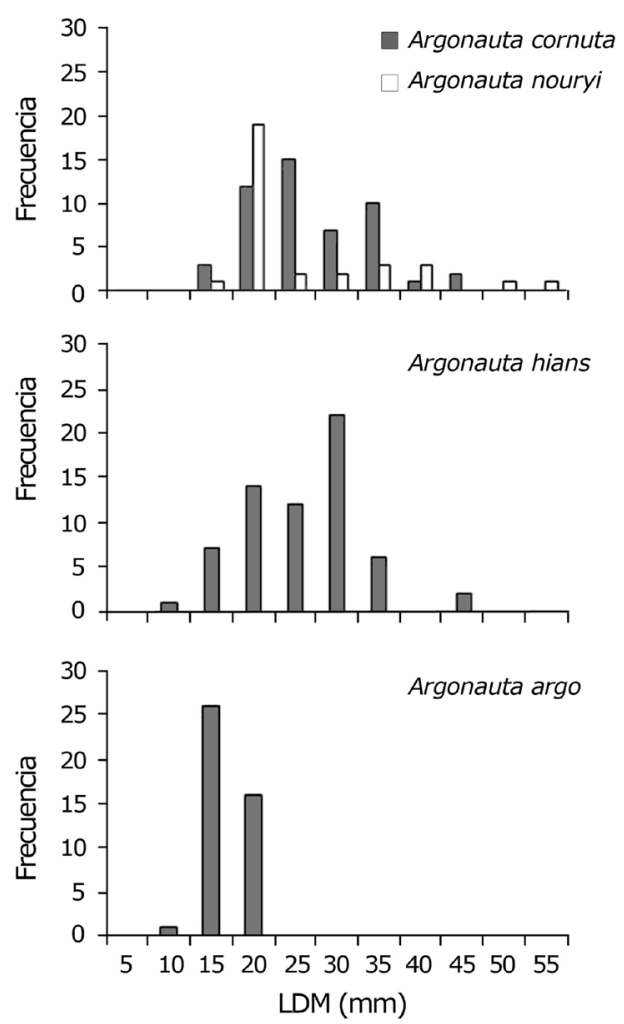

Figura 3. Distribución de tallas para las especies de argonautas en el Pacífico sur de México / Size distribution for argonaut species in the southern Pacific of Mexico
Tanto en la temporada de lluvias como en la de secas, $A$. argo, $A$. hians y $A$. nouryi estuvieron presentes en la dieta del pez vela, dorado y barrilete. En el tiburón sedoso únicamente se registró $A$. nouryi en noviembre (final de la temporada de lluvias) (Fig. 4).

Para $A$. hians y las formas de $A$. nouryi (A. cornuta y $A$. nouryi) se presentan las relaciones entre las dimensiones del pico y el tamaño de la hembra (PT y LDM), para reconstruir la masa corporal y longitud del argonauta desde el tamaño del pico. Así, mismo se incluyen fotos del pico de los argonautas encontrados en los contenidos gástricos analizados (Tabla 3).

La ecuación potencial PT-LDM para $A$. nouryi fue expresada como: $\mathrm{PT}=0,0007 \mathrm{LDM}^{2,66}, \mathrm{R}^{2}=0,82$ (t-test, $P$ $<0,05)$; para $A$. hians: $\mathrm{PT}=0,0002 \mathrm{LDM} 2,97, \mathrm{R}^{2}=0,81$ (t-test, $P<0,05)$. Ambas especies mostraron un crecimiento alométrico negativo, con un crecimiento más rápido en longitud que en peso (Fig. 5). Para A. argo no se reportan datos de peso debido a que los organismos presentaron daños en parte de los brazos.

Por otro lado, en 5 estómagos de pez vela se presentaron hembras de $A$. argo en grupos de 6, 8 y 14 organismos por estómago. Estas hembras se encontraron inmaduras y sin concha, además no se encontró hectocotilos sobre las branquias. En todos los casos, las hembras presentaron bajo grado de digestión, lo que sugiere que fueron consumidas juntas. 


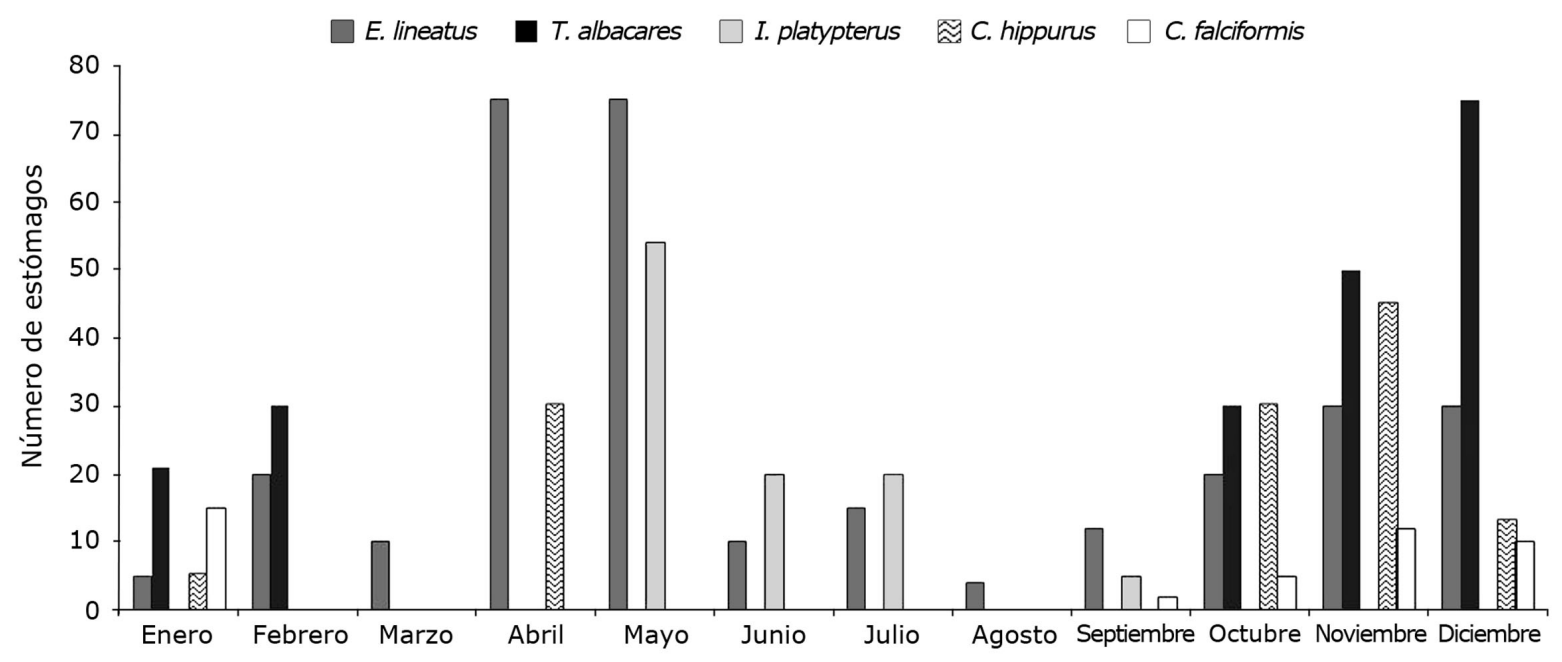

Figura 4. Número de estómagos por mes y pez depredador / Number of stomachs per month and predatory fish

Tabla 3. Ecuaciones usadas para reconstruir peso y talla del cuerpo a partir del tamaño del pico (UHL) para cada especie/ Equations used to reconstruct weight and body size from the peak size (UHL) for each species

\begin{tabular}{|c|c|c|c|c|}
\hline Especie & Mandíbula & Ecuación & $\mathrm{N}$ & $\mathrm{R}^{2}$ \\
\hline \multirow[t]{3}{*}{ Argonauta nouryi } & Superior & $\mathrm{LDM}=6,47 \mathrm{UHL}-9,03$ & 18 & 0,80 \\
\hline & & $\mathrm{Ln} \mathrm{PT}=0,20 \mathrm{Ln} \mathrm{UHL}+1,4$ & 18 & 0,84 \\
\hline & Inferior & & & \\
\hline \multirow[t]{4}{*}{ Argonauta cornuta } & Superior & $\mathrm{LDM}=4,05 \mathrm{UHL}+6,76$ & 21 & 0,86 \\
\hline & & Ln $\mathrm{PT}=1,83 \mathrm{Ln} \mathrm{UHL}-1,56$ & 21 & 0,81 \\
\hline & Inferior & $\mathrm{LDM}=11,9 \mathrm{LHL}-1,92$ & 16 & 0,83 \\
\hline & & Ln PT= 2,4 Ln LHL - 0,76 & 16 & 0,83 \\
\hline \multirow[t]{2}{*}{ A. cornuta $+A$. nouryi } & Superior & $\mathrm{LDM}=4,45 \mathrm{UHL}+3,06$ & 39 & 0,80 \\
\hline & & Ln PT $=2,15$ Ln UHL - 2,08 & 39 & 0,84 \\
\hline \multirow[t]{4}{*}{ Argonauta hians } & Superior & $\mathrm{LDM}=5,03 \mathrm{UHL}+5,03$ & 32 & 0,82 \\
\hline & & Ln PT= 2,34 Ln UHL - 2,14 & 32 & 0,80 \\
\hline & Inferior & $\mathrm{LDM}=12,82 \mathrm{LHL}-0,47$ & 32 & \\
\hline & & $\operatorname{Ln} \mathrm{PT}=3,14 \mathrm{Ln} \mathrm{LHL}-1,20$ & 32 & 0,81 \\
\hline Argonauta argo* & Inferior & $\mathrm{Ln} \mathrm{PT}=1,18 \mathrm{Ln} \mathrm{LHL}-0,55$ & 10 & \\
\hline
\end{tabular}

*Tomada de Romeo et al. (2011), Ln: logaritmo natural, LDM: longitud dorsal del manto (mm), LHL: longitud del capuchón en la mandíbula inferior $(\mathrm{mm})$, UHL: longitud del capuchón en la mandíbula superior $(\mathrm{mm})$ 


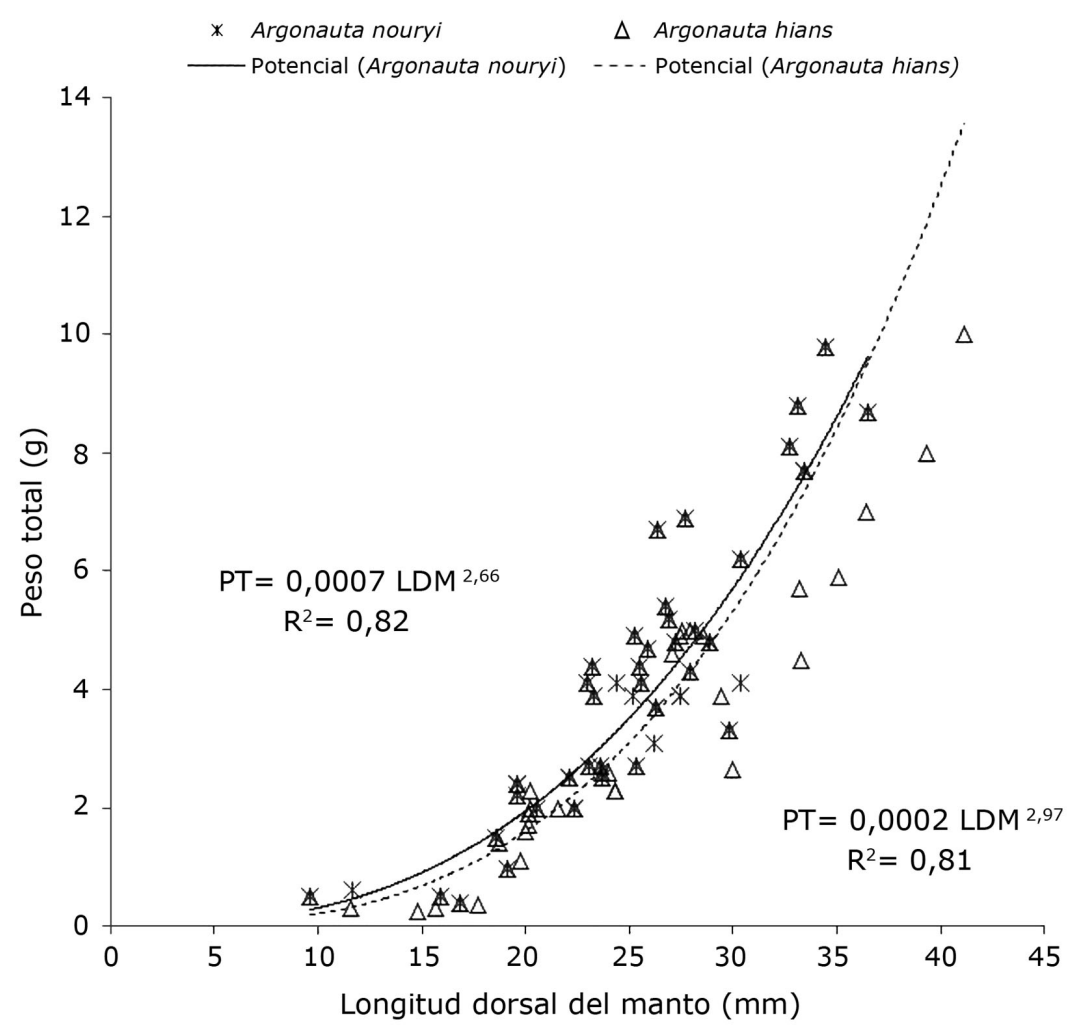

Figura 5. Relación peso total vs longitud dorsal del manto para Argonauta nouryi y Argonauta hians en el Pacífico sur de México / Relationship between dorsal length mantle vs total weight to Argonauta nouryi and Argonauta hians from Pacific south of Mexico

En estómagos de pez vela y de barrilete se observaron grupos de 5 hasta 18 hembras grávidas (concha con huevos) de $A$. nouryi en mínimo estado de digestión. Estas hembras estaban maduras y tenían suspendidos dentro de la concha huevos con un diámetro entre 0,6-1,0 mm LDM, 0,81 $\pm 0,08$ mm y embriones (0,8-1,2 mm LDM, 0,93 $\pm 0,1 \mathrm{~mm})$ en 5 diferentes estados de desarrollo de acuerdo a Naef (1923), lo que sugiere un desove continuo.

A su vez, hembras con concha de $A$. hians se presentaron en grupos de 5 a 12 hembras en estómagos de pez vela; sobre las branquias se observó de 1 a 3 hectocotilos por hembra y filamentos de penes insertados en el ovario. Las conchas presentaron huevos suspendidos, con un diámetro entre 0,7 a 1,2 mm LDM, 0,75 $\pm 0,12 \mathrm{~mm}$ y embriones en diferentes estados de desarrollo (0,7-1,3 mm LDM, 0,91 $\pm 0,08 \mathrm{~mm})$. En mayo y noviembre, estómagos de barrilete y pez vela contenían 9 hembras pequeñas de $A$. hians (6,2 a 10,4 mm LDM). Estas se encontraban inmaduras y sin concha, y con un hectocotilo sobre cada branquia. La carencia de concha en hembras con tallas menores a los $19 \mathrm{~mm}$ de LDM puede indicar que se trata de hembras inmaduras que aún no han generado su concha.

\section{Discusión}

En la última década diversos trabajos han puntualizado la importancia de los argonautas en la red trófica pelágica (Arizmendí-Rodríguez et al. 2006, Abitia-Cárdenas et al. 2010, Galvan-Magaña et al. 2013, Olson et al. 2014) y su frecuencia de ocurrencia en la dieta de numerosas especies de peces pelágicos sugiere que los argonautas son ampliamente disponibles y abundantes (Abitia-Cárdenas et al. 2010, Staudinger et al. 2013).

En la dieta de los peces analizados en este trabajo, los argonautas fueron la presa más frecuente entre los pulpos y el segundo grupo de cefalópodos más importante después de los calamares, principalmente D. gigas. Con todo, están notablemente ausentes en las prospecciones de la región (Sánchez 2003), posiblemente porque estos son capaces de evitar su captura por los métodos de muestreo tradicionales o los depredadores se alimentan selectivamente de ellos. Al respecto, el pez vela, el dorado y los túnidos son conocidos como forrajeros en la zona epipelágica (Staudinger et al. 2013), a lo anterior se añade que las hembras grávidas de argonauta forman agregaciones de varios organismos y se alimentan cerca de la superficie de presas planctónicas 
(Nesis 1977), siendo a su vez presas muy visibles y vulnerables a los depredadores pelágicos tope (Rosa \& Seibel 2010, Staudinger et al. 2013). Los resultados sugieren un comportamiento de agregación para el desove de $A$. hians y $A$. nouryi.

Cabe destacar que los depredadores oportunistas como el dorado, el tiburón sedoso y el atún aleta amarilla se alimentan de una gran variedad de presas. Al respecto, Ruíz-Pérez et al. (2016) mencionan 71 tipos de presas; sin embargo, dependen de la disponibilidad y abundancia de las mismas (Olson \& Galván-Magaña 2002, Duffy et al. 2015). El pez vela es más selectivo, consume un mayor porcentaje de cefalópodos y peces (Arizmendi-Rodríguez et al. 2006, Hernández-Aguilar et al. 2012). Así, estos depredadores pueden servir como indicadores de los cambios latitudinales en la composición e importancia de las presas, además de completar registros de especies que no son importantes para la pesca o bien que no son capturadas por los equipos de pesca convencionales (Staudinger et al. 2013), tal es el caso de los argonautas. Al respecto, en trabajos previos (Cherel et al. 2004, Arizmendi-Rodríguez et al. 2006, Claerke et al. 2006, Staudinger et al. 2013, Alejo-Plata et al. 2014, Ortega-García et al. 2018) se señala el valor de usar a los depredadores tope como "muestreadores biológicos" para obtener información sobre la distribución, abundancia y diversidad de los cefalópodos.

En la última revisión sobre la biogeografía de la familia Argonautidae, se remarca la ausencia de Argonauta hians en el Pacífico oriental (Finn 2013, 2014). Sin embargo, ha sido reportada en el sureste del California (Abbott 1968), sureste del Golfo de California (Stadum \& Saul 2000), centro del Pacífico mexicano (Hoyle 1904), Perú (Paredes et al. 1999) y Chile (Rocha 1997). En los contenidos gástricos analizados en este trabajo, $A$. hians fue la especie de argonauta más abundante $(33,6 \%)$, y se encontró en un mínimo estado de digestión, lo que sugiere que fueron consumidas en la zona de estudio. Considerando los reportes anteriores sobre su distribución y los resultados de este trabajo, se cuenta con evidencia suficiente para reconocer la presencia de $A$. hians en el Pacífico oriental.

Por otro lado, el músculo blando de los cefalópodos es digerido rápidamente, quedando sólo los picos como único indicio de su consumo (Galván-Magaña et al. 2013). En peces pelágicos, la tasa metabólica puede ser hasta 3 veces más alta que en otras especies de peces (Ware 1975); por ejemplo, la duración de la digestión en $C$. hippurus y $T$. albacares fue de 6 a 18 h (Olson \& Galván-Magaña 2002). Considerando que las conchas de los argonautas son muy frágiles y que se encontraron hembras con las conchas intactas, aún llenas de huevos y embriones, sugiere que fueron consumidas en la zona de estudio y muy cerca de la zona de pesca de las embarcaciones artesanales.
Así mismo, la identificación de los argonautas en los estómagos de depredadores ha presentado problemas debido a que generalmente no se encuentran las conchas completas. La identificación hasta especie basada en los picos es difícil por la carencia de claves ilustradas; debido a esto, la mayoría de los trabajos los reporta como Argonauta spp. Los resultados de este trabajo proporcionan información para diferenciar los picos de A. argo, A. hians y A. nouryi. Los picos son un buen medio para identificar especies de argonautas siendo la mandíbula inferior la estructura más adecuada para separar a las especies; esto debido a que presenta características más conspicuas, como es la forma del rostro y nivel de pigmentación.

En cuanto a los machos de argonauta, tienen una longitud del manto de no más de $2 \mathrm{~cm}$ (1:12 la longitud del manto de la hembra) y nunca producen concha, habitan en el ambiente pelágico (Nesis 1977) y forman parte del micro necton (Vidal et al. 2010); no se tienen reportes de ser presa de peces pelágicos. Sin embargo, tienen un brazo hectocotilizado muy desarrollado que permanece encerrado en un saco bajo el ojo izquierdo y durante la cópula se desprende (Guerra 1992). La presencia de hectocotilos en la cavidad del manto de pequeñas hembras de $A$. hians $(\mathrm{LDM}=11 \mathrm{~mm})$ sugiere apareamiento a la edad temprana, esto también ha sido observado en Argonauta boettgeri por (Nessis 1977) y Argonauta nodosa (Vidal et al. 2010). Estos autores sugieren que la copulación tiene lugar cuando los machos y hembras son de tamaño similar y habitan el ambiente pelágico. Así, el crecimiento alométrico observado en $A$. nouryi y $A$. hians se puede deber a la madurez sexual anticipada y a que son desovadores continuos (Nessis 1977).

Adicionalmente, Vidal et al. (2010) reportaron que los machos y hembras jóvenes y sin concha de $A$. nodosa se encuentran asociados a sitios poco profundos de surgencia, y con elevadas concentraciones de clorofila- $a$. En la zona de estudio, de noviembre hasta abril se registran temperaturas en la superficie del mar relativamente bajas $\left(\sim 17^{\circ} \mathrm{C}\right)$ y valores por arriba de 1,0 mg de clorofila- $a$ asociados a los Tehuanos, esto podría explicar la presencia de hembras pequeñas inmaduras carentes de concha en los estómagos del barrilete y pez vela en noviembre y diciembre.

Guerra et al. (2002) evidenciaron que A. argo es un organismo poiquilotermo; por su parte, Ortega-García et al. (2018) registraron la presencia de Argonauta spp. en estómagos marlín rayado Tetrapturus audax, recolectados en meses cálidos y fríos y concluyen que las especies del género Argonauta pueden tolerar cambios en la temperatura superficial del mar. En la zona de estudio, A. argo se presentó al inicio de la temporada de lluvias con la temperatura superficial del mar por arriba de $28,5^{\circ} \mathrm{C}$; Mientras que $A$. nouryi, $A$. cornuta y $A$. hians se encontraron tanto en temporada de lluvias como en secas, con temperaturas de la superficie del mar relativamente bajas $\left(\sim 17^{\circ} \mathrm{C}\right)$. 
Asimismo, los argonautas son considerados indicadores útiles de condiciones oceanográficas inusuales, así como de las tendencias del calentamiento global (Guerra et al. 2002). Sus hábitos de vida pelágica los hacen particularmente vulnerables a la acidificación del océano, y la composición de calcita-Mg de la concha es susceptible de disolución a medida que el océano se calienta y disminuye el $\mathrm{pH}$ (Wolfe et al. 2012), aunado a que su concha carece de protección por mucosas o epitelio de recubrimiento, y tiene una mayor proporción área-volumen de exposición (Smith et al. 2012). Para la zona de estudio, Chapa-Balcorta et al. (2015) observaron cerca de la superficie del mar y después de un evento Tehuano, valores de saturación del carbonato de calcio en forma de aragonita y de $\mathrm{pH}$ tan bajos como 1,1 y 7,5, respectivamente. Para estos autores, la presencia de arrecifes de coral y otros organismos calcificadores (como los argonautas) en la región, plantea algunas preguntas: ¿Cómo sobreviven en dichas condiciones? ¿Los organismos del Golfo de Tehuantepec han desarrollado estrategias adaptativas para sobrevivir?

Los resultados de este trabajo indican la presencia de una población importante de $A$. hians y $A$. nouryi en el área de estudio. Un desove continuo y apareamiento a la edad temprana, parece constituir una estrategia efectiva de estas especies para sobrevivir en el Golfo de Tehuantepec.

Si bien, el estudio de los cefalópodos en los contenidos estomacales de depredadores obtenidos de la pesca artesanal proporciona información invaluable sobre los ecosistemas locales, también presenta varias limitantes: i) Los peces depredadores muestreados en este trabajo, son migratorios; al respecto, Markaida \& Hochberg (2005) mencionan que en los peces migratorios la distancia entre su última comida y su captura es desconocida. ii) El acceso a los depredadores está limitado a su presencia estacional en la región; además, en México la pesca de dorado y pez vela se encuentra restringida a la pesca deportiva, los peces analizados en el presente estudio forman parte de las capturas incidentales de la pesca artesanal. En el caso del tiburón, existe un periodo de veda de mayo a julio. iii) La distancia que pueden cubrir las embarcaciones de pesca artesanal se restringe al borde de la plataforma continental, a no más de 30 millas de la línea de costa.

En este trabajo queda de manifiesto la utilidad de los estudios de alimentación sobre peces depredadores pelágicos, como método alternativo para el estudio de la distribución y biología de los argonáutidos, siendo el primer estudio para las especies del Pacífico tropical oriental. Además, los resultados recalcan la importancia de los argonautas en las redes alimenticias estacionales, y la necesidad de más investigaciones sobre la identidad de las especies de argonautas usando herramientas moleculares.

\section{Agradecimitentos}

Este estudio fue apoyado por el CONACyT Convocatoria Problemas Nacionales (2015-1740), proyecto "Calamares: Recurso pesquero alterativo para la costa de Oaxaca". Agradecemos a los pescadores artesanales de Oaxaca por su apoyo en la obtención de muestras, a Daniel Armengol y Ezequiel Rodríguez por su ayuda en el trabajo de campo y a Oscar Illescas por las fotografías de los picos de argonauta. Agradecemos y apreciamos la revisión crítica de los árbitros anónimos y Editora de la RMBO Claudia A. Bustos. MCAP agradece al programa SNI-CONACyT.

\section{LiTERATURA CITADA}

Abbott RT. 1968. Seashells of North America: a guide to field identification, 280 pp. Golden Press, New York.

Abitia-Cárdenas LA, D Arizmendi-Rodríguez, N GudiñoGonzález \& F Galván-Magaña. 2010. Feeding of blue marlin Makaira nigricans off Mazatlan, Sinaloa, Mexico. Latin American Journal of Aquatic Research 38: 281-285.

Arizmendi-Rodríguez DI, LA Abitia-Cárdenas, F GalvánMagaña \& I Trejo-Escamilla. 2006. Food habits of sailfish Istiophorus platypterus of Mazatlan, Sinaloa, México. Bulletin of Marine Science 79(3): 777-791.

Cabrera-Chávez-Costa A, F Galván-Magaña \& O EscobarSánchez. 2010. Food habits of the silky Carcharhinus falciformis (Müller \& Henle, 1839) off the western coast of Baja California Sur, México. Journal of Applied Ichthyology 26: 499-503.

Chapa-Balcorta C, JM Hernández-Ayón, R Durazo, E Beier, SR Alin \& A López-Pérez. 2015. Influence of post-Tehuano oceanographic processes in the dynamics of the $\mathrm{CO}_{2}$ system in the Gulf of Tehuantepec, Mexico. Journal of Geophysical Research, Oceans 120(12): 7752-7770.

Clark MR. 1986. A handbook for the identification of cephalopod beaks, 273 pp. Clarendon Press, Oxford.

Duffy L, RJ Olson, CE Linnet-Cody, F Galván-Magaña, N Bocanegra-Castillo \& PM Kuhnert. 2015. Foraging ecology of silky sharks, Carcharhinus falciformis, captured by the tuna purse-seine fishery in the eastern Pacific Ocean. Marine Biology 162(3): 571-593.

Finn JK. 2013. Taxonomy and biology of the argonauts (Cephalopoda: Argonautidae) with particular reference to Australian material. Molluscan Research 33: 143-222.

Finn JK. 2014. Family Argonautidae. In: Jereb P, CFE Roper, MD Norman \& JK Finn (eds). Cephalopods of the world. An annotated and illustrated catalogue of cephalopod species known to date. Volume 3. Octopods and Vampire Squids, pp. 228-236. FAO Species Catalogue for Fishery Purposes, Rome.

Galván-Magaña F, C Polo-Silva, SB Hernández-Aguilar, A Sandoval-Londoño, MR Ochoa-Díaz, N AguilarCastro, D Castañeda-Suárez, A Cabrera-Chávez-Costa, A Baigorrí-Santa Cruz, E Torres-Rojas \& LA AbitiaCárdenas. 2013. Shark predation on cephalopods in the Mexican and Ecuadorian Pacific Ocean. Deep-Sea Research II 95: 52-62. 
Guerra A. 1992. Mollusca Cephalopoda. En: Ramos ME (ed). Fauna Ibérica 1: 1-327. Museo Nacional de Ciencias Naturales, Consejo Superior de Investigaciones Científicas, Madrid.

Guerra A, AF Gonzalez \& F Rocha. 2002. Appearance of the common paper nautilus Argonauta argo related to the increase of the sea surface temperature in the north-eastern Atlantic. Journal of the Marine Biological Association of the United Kingdom 82: 855-858.

Hernández-Aguilar SB, A Abitia-Cárdenas, XG MorenoSánchez, M Arellano-Martínez \& E González-Rodríguez. 2012. Trophic spectrum of the sailfish Istiophorus platypterus caught off Acapulco in the southern Mexican Pacific. Journal of the Marine Biological Association of the United Kingdom 93(4): 1097-1104.

Hoyle WE. 1904. Reports on the dredging operations off the west coast of Central America to the Galapagos, to the west coast of Mexico, and in the Gulf of California, in charge of Alexander Agassiz, carried on by the U. S. Fish Commission steamer "Albatross" V. Reports on the Cephalopoda. Bulletin of the Museum of Comparative Zoology at Harvard 43: 1-72.

Lluch-Cota SE, S Álvarez-Borrego, EM Santamaría-del Ángel, FE Müller-Karger \& S Hernández-Vázquez. 1997. El Golfo de Tehuantepec y áreas adyacentes: variación espacio temporal de pigmentos fotosintéticos derivados de satélite. Ciencias Marinas 23(3): 329-340.

Markaida U \& FG Hochberg. 2005. Cephalopods in the diet of swordfish (Xiphias gladius) caught off the west coast of Baja California, Mexico. Pacific Science 59(1): 25-41.

Markaida U \& O Sosa-Nishizaki. 2010. Food and feeding habits of the blue shark Prionace glauca caught off Ensenada, Baja California, México, with a review on its feeding. Journal of the Marine Biological Association of the United Kingdom 90: 1-18.

Nesis KN. 1977. The biology of paper nautilus, Argonauta boettgeri and Argonauta hians (Cephalopoda, Octopoda) in the Western Pacific Ocean and the seas of the East Indian Archipelago. Zoologicheskii Zhurnal 56(7): 1004-1014.

Olson RJ \& F Galván-Magaña. 2002. Food habits and comparison rates of common dolphinfish (Coryphaena hippurus) in the eastern Pacific Ocean. Fishery Bulletin 100: 279-298.

Olson RJ, LM Duffy, PM Kuhnert, F Galván-Magaña, N Bocanegra-Castillo \& V Alatorre-Ramírez. 2014. Decadal diet shift in yellow tuna Thunnus albacares suggests broadscale food web changes in the eastern tropical Pacific Ocean. Marine Ecology Progress Series 497: 157-178.

Ortega-García S, DI Arizmendi-Rodríguez \& M ZúñigaFlores. 2018. Striped marlín (kajikia audax) diet variability off Cabo San Lucas, B.C.S., Mexico during El Niño-La Niña events. Journal of the Marine Biological Association of the United Kingdom 98(6): 1513-1524. < doi:10.1017/ S0025315417000595>

Osuna-Peralta YR, D Voltolina \& RE Morán-Angulo. 2014. Stomach contents of the Pacific Sharpnose shark, Rhizoprionodon longurio (Carcharhiniformes, Carcharninidae) in the Southeastern Gulf of California. Latin American Journal of Aquatic Research 42(3): 438-444.
Paredes C, P Huamán, F Cardoso, R Vivar \& V Vera. 1999. Present state of the knowledge of aquatic mollusks in Peru. Revista Peruana de Biología 6: 5-47.

Reyes S \& A Mejía-Trejo. 1991. Tropical perturbations in the eastern Pacific and the precipitation field over northwestern Mexico in relation to ENSO phenomenon. International Journal of Climatology 11: 515-528.

Rocha F. 1997. Cephalopods in Chilean waters, a review. Malacological Review 30: 101-113.

Romero-Centeno R, J Zavala-Hidalgo, A Gallegos \& JO'Brien. 2003. Isthmus of Tehuantepec Wind Climatology and ENSO Signal. Journal of Climate 16: 2628-2639.

Rosa R \& BA Seibel. 2010. Voyage of the argonauts in the pelagic realm: physiological and behavioural ecology of the rare paper nautilus, Argonauta nouryi. ICES Journal of Marine Science 67: 1494-1500.

Rosas-Alayola J, A Hernández-Herrera, F Galván-Magaña, LA Abitia-Cárdenas \& AF Muhlia-Melo. 2002. Diet composition of Sailfish (Istiophorus platypterus) from the southern Gulf of California, Mexico. Fisheries Research 57: 185-195.

Ruíz-Pérez N, G Cerdenares-Ladrón de Guevara, D LópezHerrera \& R Altamirano. 2016. Relaciones tróficas entre cinco especies de peces pelágicos que cohabitan en las costas de Oaxaca, México. Hidrobiológica 26(1): 77-85.

Salcedo-Vargas MA. 1991. Checklist of the cephalopods from the Gulf of Mexico. Bulletin of Marine Science 49: 216-220.

Sánchez P. 2003. Cephalopods from off the Pacific coast of Mexico: biological aspects of the most abundant species. Scientia Marina 67(1): 81-90.

Smith AM, K Wolfe \& M Byrne. 2012. Argonauta at risk: dissolution and carbonate mineralogy of egg cases. In: Yellowlees D \& TP Hughes (eds). Proceeding of the $12^{\text {th }}$ International Coral Reef Symposium, Cairns, Australia, 9-13 July 2012, Organism and ecosystem responses to ocean acidification, Paper 8A-1: 4 pp. James Cook University, Cairns. $<$ http://www.icrs2012.com/proceedings/ manuscripts/ICRS2012_8A_1.pdf $>$

Stadum CJ \& LR Saul. 2000. Argonauts of the late Miocene, Los Angeles Basin, southern California. Annual Report Western Society Malacologist 32: 47-50.

Staudinger MD, F Juanes, B Salomon \& AK Teffer. 2013. The distribution, diversity, and importance of cephalopods in top predator diets from offshore habitats of the Northwest Atlantic Ocean. Deep-Sea Research II 95: 182-192.

Trasviña A, ED Barton, J Brown, HS Vélez, PM Kosro \& RL Smith. 1995. Offshore wind forcing in the Gulf of Tehuantepec, Mexico: The asymmetric circulation. Journal of Geophysical Research 100(C10): 20649-20663.

Tripp-Valdez A, F Galván-Magaña \& S Ortega-García. 2010. Feeding habits of dolphinfish (Coryphaena hippurus) in the southeastern Gulf of California, Mexico. Journal Applied Ichthyology 26(4): 578-582.

Vidal E, M Haimovici \& VCS Hackbart. 2010. Distribution of paralarvae and small juvenile cephalopods in relation to primary production in an upwelling area off southern Brazil. ICES Journal of Marine Science 67: 1346-1352. 
Ware DM. 1975. Growth, metabolism, and optimal swimming speed of a pelagic fish. Journal of Fisheries Research Board of Canada 32(1): 33-41.

Wolfe K, AM Smith, P Trimby \& M Byrne. 2012. Vulnerability of the paper nautilus (Argonauta nodosa) shell to a climate change ocean: potential for extinction by dissolution. Biological Bulletin 223: 236-244.
Xavier JC \& Y Cherel. 2009. Cephalopod beak guide for the Southern Ocean, 129 pp. British Antarctic Survey, Cambridge.

Recibido el 19 de julio de 2018 y aceptado el 24 de enero de 2019

Editor: Claudia Bustos D. 